Review

\title{
Spotlight on Older Adults in Swallowing Research
}

Marie Jardine *, Anna Miles, Jacqui Allen

University of Auckland, Building 507, 22-30 Park Ave, Grafton, Auckland, New Zealand; E-Mails: m.jardine@auckland.ac.nz; a.miles@auckland.ac.nz; jeallen@voiceandswallow.co.nz

* Correspondence: Marie Jardine; E-Mail: m.jardine@auckland.ac.nz

Academic Editor: David G Smithard

Collection: Dysphagia in the Elderly

\section{OBM Geriatrics}

2020, volume 4, issue 4

doi:10.21926/obm.geriatr.2004146
Received: Novenber 07, 2020

Accepted: December 11, 2020

Published: December 25, 2020

\begin{abstract}
Generalisations and misconceptions about ageing and swallowing can influence clinical opinion. Assumptions about swallowing function based on someone's age risk overmedicalising or, conversely, undertreating older patients. Evidence suggests that increased prevalence of swallowing problems in older age is attributed to pathology known to affect swallowing function, or an unrelated serious illness and combination of health factors, and not ageing itself. This review highlights factors clinicians should consider when interpreting studies involving older adults and recommends methodological parameters for those researching swallowing with older participants.
\end{abstract}

\section{Keywords}

Deglutition; deglutition disorders; aged, 80 and over

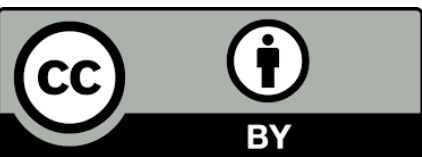

(C) 2020 by the author. This is an open access article distributed under the conditions of the Creative Commons by Attribution License, which permits unrestricted use, distribution, and reproduction in any medium or format, provided the original work is correctly cited. 


\section{Introduction}

Older age does not define our physical and mental abilities; some adults in their 80s have similar capacities to 20-year-olds [1]. However, our bodies do change with age. A hallmark of retirement is leisure which typically features food, drink, and socialising. For many, eating patterns may be altered by dentition, such as missing teeth, dental protheses, or dentures [2]. The effects of age on swallowing are less obvious as they cannot be visualised without instrumental assessment. Understanding swallowing function in older age has greatly improved thanks to advancements in technology and analysis [3-5]. Quantitative measures have enhanced the accuracy and reliability of interpretation of high resolution impedance manometry and videofluoroscopy [6]. Healthy ageing may involve anatomical changes, such as spinal (e.g. osteophyte) and a non-obstructive cricopharyngeal bar [7]. Swallowing physiology remains functional; the airway is protected and aspiration is a rare event $[8,9]$. Consistently reported age effects include increased swallow reaction, bolus transit, and UES opening times, and pressure changes at the UES and LES $[8,10,11]$. Swallowing compensatory strategies may be spontaneously adopted, such as dose metering (dividing a bolus in the mouth so that the quantity swallowed is smaller), clearing oral residue, and taking smaller sips or mouthfuls [12].

Any swallowing behaviours that compromise respiratory health or nutritional intake are indicative of dysphagia, such as repeated coughing during mealtimes, nasal redirection, and regurgitation [13]. Swallowing problems negatively impact on what should be enjoyed in retirement, contributing to social isolation and caregiver burden $[14,15]$. Consequences may include unintended weight loss; an inability to eat in public; prolonged mealtimes; difficulty swallowing liquids, solids, or pills; pain when swallowing; reduced enjoyment when eating or drinking; food sticking; coughing; and stress when swallowing [16]. The increased prevalence of swallowing impairment in older age and forecast of ageing populations prioritise research that improves dysphagia management and outcomes for older adults and their support networks. Timing, displacement, area, and pressure measures offer a more direct comparison across ages and between individuals with and without swallowing problems (dysphagia) [17], with the potential to predict risk, such as aspiration [18]. However, generalisations and misconceptions about ageing and swallowing continue to arise. Literature without a neutral position risks influencing clinical opinion.

Underrepresentation of very old adults can also limit the clinical application of research findings. The 5Ts framework (target population, team, tools, time, and tips) was recently developed to address barriers to recruitment of older adults in clinical research and provide a strategy for inclusion [19]. This framework complements the $5 \mathrm{Ms}$ (mind, mobility, medications, multicomplexity, and matters most to me) which promotes the key competencies of geriatrics in clinical practice [20]. With reference to these frameworks (Figure 1), this review highlights factors clinicians should consider when interpreting swallow studies with older adults, and recommends methodological parameters for those researching swallowing with older participants. 


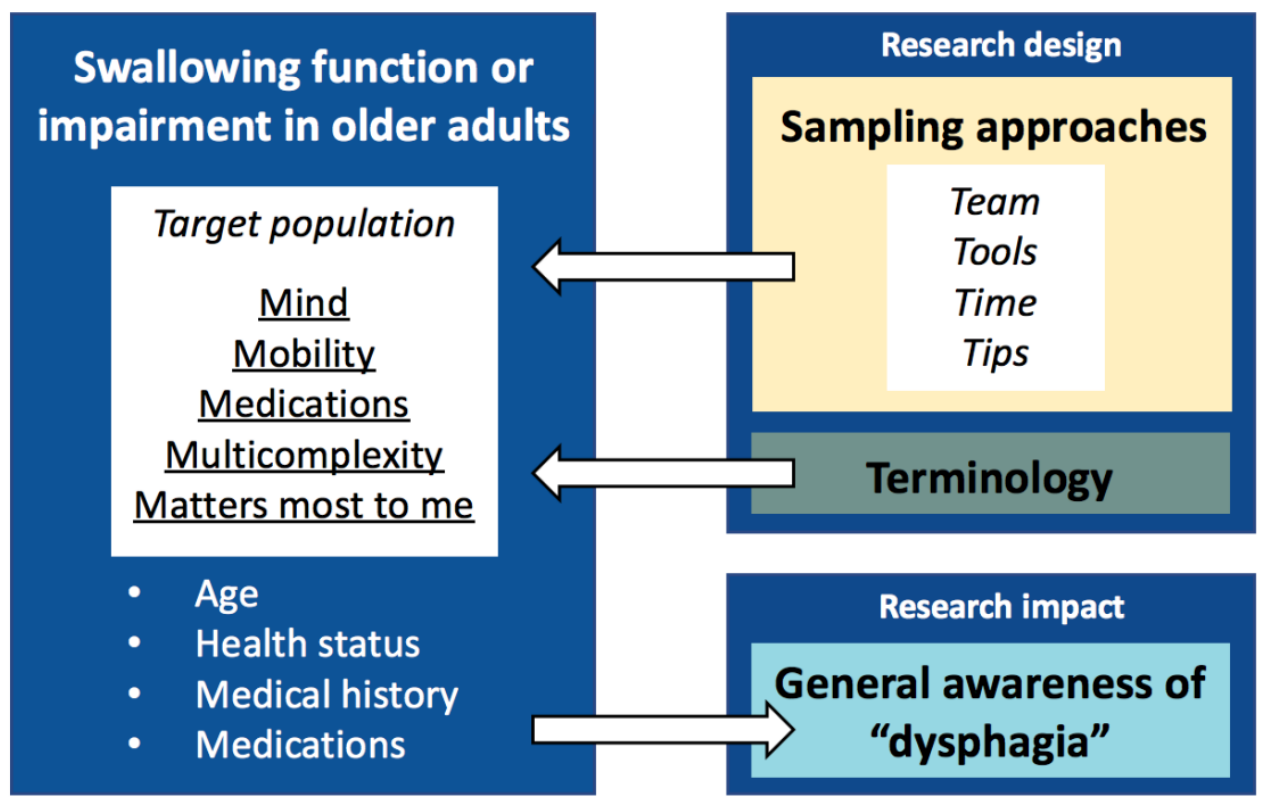

Figure 1 Factors to Consider in Swallowing Research with Older Adults (5Ts[19] and $5 \mathrm{Ms}[20])$.

\section{Swallowing Function and Impairment in Older Adults}

Swallowing problems are a symptom; there is an underlying cause (Figure 2). Depending on the aetiology, symptoms may develop gradually or occur suddenly. Progressive swallowing symptoms may foreshadow pathology (e.g., head and neck or oesophageal cancer) or eventuate in the later stages of a disease (e.g., motor neurone disease, dementia, or Parkinson's disease). The severity of swallowing difficulties depends on the stage or prognosis of diagnosis, neurological involvement, health status, comorbidities, and medications. For older adults without a specific diagnosis or history of swallowing impairment but who are battling a serious illness, lower functional reserve, comorbidities, and medications may cause swallowing problems. A retrospective study compared videofluoroscopic swallow studies of healthy older adults with older adults hospitalised for a reason unrelated to swallowing, who developed swallowing problems during their stay [17]. Patients demonstrated reduced pharyngeal constriction, which was associated with the impact of illness and hospitalisation, and not age. 


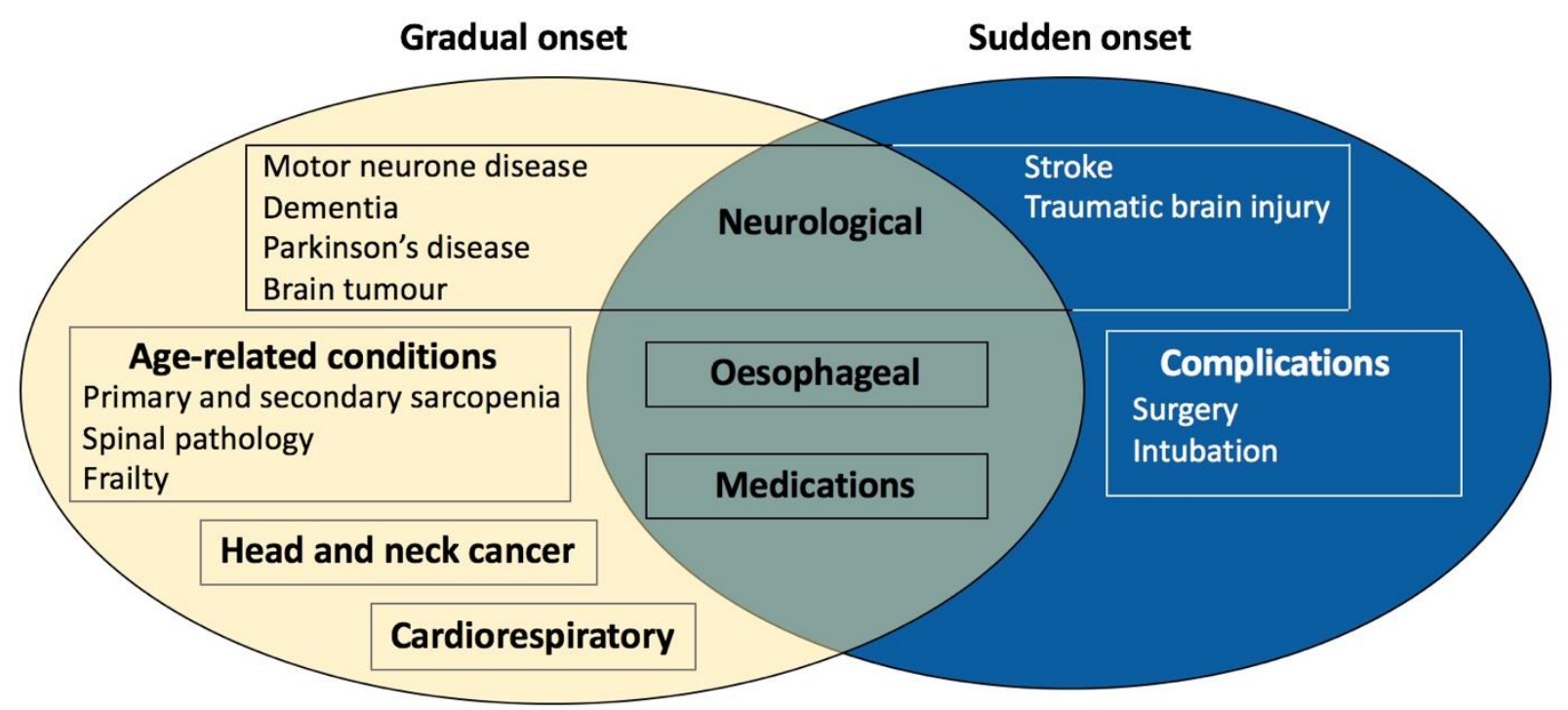

Figure 2 Commonly Reported Causes of Swallowing Impairment in Older Age.

The notion that ageing itself does not cause swallowing problems is echoed throughout the literature but can get overshadowed or confused by the increasing dysphagia prevalence in the ageing population. Older adults are a heterogeneous group, therefore swallowing function does not follow a single trajectory over time. Figure 3 proposes four models of swallowing function and impairment in older age. A and B represent normal age-related swallowing changes which do not affect overall function or safety [21]. Healthy adults may not encounter changes in swallowing function (A) or unknowingly adapt to slight alterations over time (presbyphagia), such as by performing swallowing compensatory behaviours (B). C and D depict a deterioration in swallowing function and safety due to a diagnosis or health condition known to affect swallowing [17]. After a period of recovery from a serious illness, swallowing symptoms may resolve, although they may not return to baseline due to the effect of increasing comorbidities and medications (C). Alternatively, swallowing problems may persist indefinitely (D) due to disease (e.g. Parkinson's disease) or medical event (e.g. stroke). In swallowing studies, it is important for clinicians and researchers to consider the swallowing function of older participants with respect to their age, health status, medical history, and medications. 


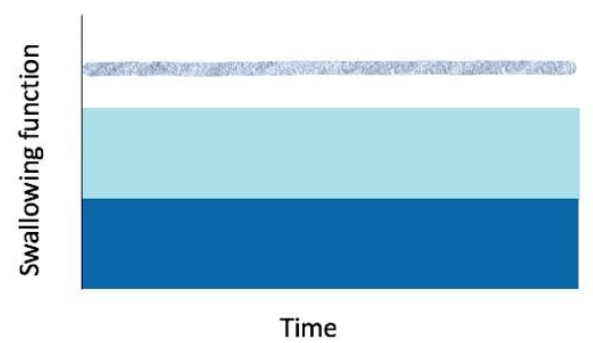

A

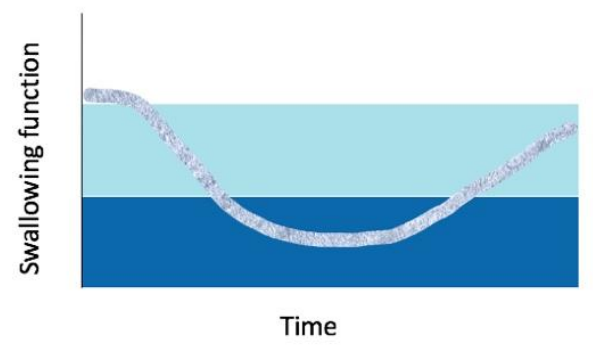

C

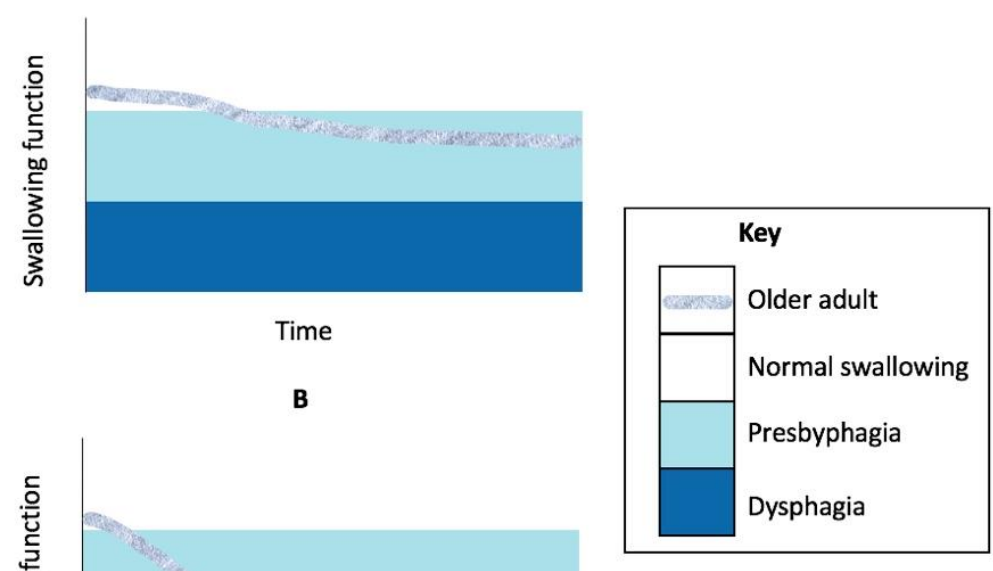

Figure 3 Four Models of Swallowing Function and Impairment in Older Age.

\subsection{Age}

With longer life expectancies, older age research has evolved into cohorts: young old (65-74 years), old old (75-84 years) and oldest old (85+ years) [22], and age bands: sexagenarians (60-69 years), septuagenarians (70-79 years, ) octogenarians (80-89 years), nonagenarians (90-99 years), and centenarians ( $>100$ years) [23]. Swallowing studies with participants in their late $80 \mathrm{~s}$ and $90 \mathrm{~s}$ are emerging. This is important to plan for the ageing of our patient population. A single site study from 2007 to 2014 observed a 222\% increase in acute dysphagia referrals for hospitalised patients 90 years and older [24]. Participant recruitment requires careful planning to ensure the oldest old are represented in countries with ageing populations, such as applying the 5Ts framework [19]. A recent systematic review on the effects of age on swallowing identified only one third of published studies (38/107) recruited adults over 85 years old [8]. Results should, however, be interpreted for one's own respective country demographics, such as life expectancy. For example, life expectancy in Cambodia or the Philippines is 15 years lower than in Japan or Hong Kong [25]. Researchers investigating ageing and swallowing should transparently report country of research and age of participants, including mean, range and standard deviation.

\subsection{Health Status, Medical History, and Medications}

Health status may include level of dependency and living situation or place of swallowing assessment (e.g. in hospital or residential care facility). Researchers should detail medical history known to affect swallowing function, as well as number of comorbidities and prescribed medications. In a survey based study, community-living older respondents with medical history or medications known to affect swallowing function presented with dysphagia risk [21] (abnormal Eating Assessment Tool (EAT-10) scores [16]). A cross-sectional study observed prolonged pharyngeal transit times in asymptomatic older adults with common medical conditions, especially hypertension, compared to those without [26]. Medication use increases in older age and 
polypharmacy is associated with adverse effects for older adults [27]. Therefore, side effects of medications on swallowing function should also be considered [28].

\section{Research Design}

It is important for clinicians to critically appraise the recruitment criteria for published studies before relating findings to a clinical setting. Researchers should use terminology carefully to avoid ambiguity and prevent misleading readers.

\subsection{Sampling Approaches}

In normative studies, participants should be recruited using validated screening assessments in order to meet healthy criteria. For example, the EAT-10 [16] is quick to administer and has been validated in a number of languages. Findings from a recent study of over 1000 community-dwelling over 65 year olds support the use of the EAT-10 with older adults [21]; scores were normal for older adults without medical history known to affect swallowing $(<3)$. The survey suggests that the selfreport of healthy older adults should be trusted and offers value in population screening. The description "asymptomatic" is ambiguous and increases the risk of bias, as it lacks supportive evidence. A frequently cited early study is titled "Altered Swallowing Function in Elderly Patients Without Dysphagia" [29]. Only a fifth of participants (9/56) demonstrated "a synchronous and symmetric swallowing function without morphodynamic abnormalities" (p. 1182). However, almost half of the participants had neurological conditions, including dementia and Parkinson's disease which likely misled results. Another landmark study investigated oesophageal motor function in adults over 90 years old [30]. Again, participants were not representative of healthy ageing; some had dementia and were bedridden. Descriptions of videofluoroscopic results included "disorganised and inefficient" (p. 1475), "defects", and "abnormalities" (p. 1478). Motility disorders have since been attributed to age-related medical problems - not age [31].

\subsection{Terminology}

Terms are used in the swallowing literature to refer to the older demographic, such as sarcopenic dysphagia [32] and presbyphagia [33]. However, there are risks associated with labels, including misuse and stigma [34]. Clinicians should primarily focus on an individual's swallowing symptoms, rather than whether they represent a generalised label. Researchers should ensure terminology is based on a reliable source, limitations are acknowledged, and the application of the definition is not overexaggerated.

Studies on how swallowing muscles can alter with age, including atrophy (e.g., geniohyoid [35, 36], pharyngeal area [37]) and strength (e.g., reduced tongue pressure [38]), discuss the role of sarcopenia: the age-related loss of skeletal muscle mass and strength that can compromise physical ability and quality of life [39]. Sarcopenia is differentiated between primary (caused by age alone) and secondary (resulting from disuse, malnutrition, and disease) [40]. There is a lack of information about the causes of sarcopenia due to challenges of definition, measurement, and modelling [41]. Likewise, the direct cause of sarcopenic dysphagia remains to be understood [42]. A position paper from Japan recently reported key criteria to prevent misuse of the term; sarcopenia must exist in 
the whole body and not be caused by neuromuscular diseases [32]. Researchers should ensure they keep up to date with revisions of definitions or criteria for terminology.

Presbyphagia is a popular term that acknowledges the non-clinical effects of age on swallowing function [33]. A seminal paper described presbyphagia as "changes in the swallow mechanism with age" (p. 391) and "subtle variation in the swallow process due to ageing"(p. 394) [43]. More recently, a study proposed that presbyphagia "may be silent and slowly progressive" (p. 443) [44]. However, there is an over-reporting of presbyphagia in clinical practice in symptomatic patients blurring the boundaries between healthy presbyphagic changes in swallowing and true dysphagia. Symptomatic swallowing difficulties should not be written off as age-related presbyphagia. If presbyphagia is exacerbated by weakness or acute illness and the older adult develops swallowing problems, this signifies a transition to swallowing impairment [33] and is not healthy ageing.

\section{Research Impact}

Education is needed to raise awareness about the causes of swallowing impairment in older age. Interprofessional and continuing development forums should emphasise that any older adult with swallowing difficulties should not be ignored. A recent study reported low levels of agreement and poor to moderate levels of accuracy among clinicians differentiating swallowing impairment from normal variability, regardless of age [45]. Clinicians working with older adults should have a comprehensive understanding of normal variance in swallowing biomechanics and on the effects of age on swallowing physiology.

\subsection{General Awareness of "Dysphagia"}

Swallowing is a natural action that normally requires little thought or effort. General knowledge of swallowing difficulties by the public is still not enough [46]. Fortunately, many older adults can perceive and report new swallowing difficulties and seek help. However, in the case of serious illness, disorientation, delirium, significant language disorder (aphasia), or cognitive impairment, older adults may not be aware of or be able to communicate their swallowing problems. Those hospitalised are at risk of presenting below their baseline function and being discharged at a lower functional level [47]. These older patients may present with new swallowing problems that can go undetected as the medical team focuses on the acute presenting illness (e.g., hip fracture or urinary tract infection). Often the new swallowing problems are not acted upon until there is a consequence, such as choking, aspiration pneumonia, dehydration, or weight loss. A recent international survey highlighted that many health facilities (62\%) do not routinely screen for swallowing difficulties, nor train their staff to perform swallow screens with older patients [48]. Regular swallow screening may alert nursing and healthcare staff to signs of swallowing difficulties before consequences eventuate. These staff play central roles in dispensing medication and facilitating mealtimes. Nurses and aged care staff should be trained by speech-language therapists to notice changes in swallow function so referrals for swallowing assessments can occur. Silent aspiration and pharyngeal residue may require detection through instrumental swallowing assessment. Clinicians and researchers should not ignore new swallowing difficulties and put them down to ageing. They should treat the symptoms within the context of managing the overarching cause of dysphagia as a multidisciplinary team. 
Older adults may have preconceived ideas of ageing which influence whether they seek medical attention [49]. Less than half (39.3\%) of adults from an independent living village were not confident about whether ageing was associated with swallowing problems and almost one quarter (23.4\%) attributed swallowing difficulties to age [50]. Just over half of community-living older adults reported no previous knowledge of the term dysphagia (58.3\%) and only a third (35.5\%) correctly defined dysphagia [21]. Concerningly, in a multinational study only $39 \%$ of adults with impaired swallowing thought they could be treated [15]. The consequences of delaying swallowing assessment depend on the onset and severity of dysphagia. For example, if symptoms caused by Zenker's diverticulum (pharyngeal pouch) are ignored by older adults, by the time medical attention is sought, surgery may be unsafe or carry significant morbidity [51]. In addition, symptoms may be the "tip of the iceberg", such as a laryngeal or pharyngeal tumour, neuromuscular disease, or disease or cancer of the gastrointestinal tract [52]. More initiatives are needed to raise awareness about the causes of swallowing problems in older age to encourage full assessment and investigation and therefore ensure early identification and management.

\section{Conclusion}

Dysphagia is not inevitable as we age. Swallowing impairment may be misconceived as associated with ageing due to the increased prevalence of swallowing difficulties in older adults. However, this increased prevalence is attributed to diseases and conditions that are more common in older age. This review highlights inconsistencies in the age-related swallowing literature, urging readers to question characteristics of older participants (age, health status, medical history, medications) and research design (sampling approaches and terminology), and not overgeneralise results. Any swallowing difficulty should be reported by older adults to health professionals who must not ignore or attribute symptoms to ageing. For older adults unable to advocate for themselves due to serious illness, cognitive impairment, or aphasia, health professionals are responsible for identifying swallowing impairment. More work is needed to prevent overmedicalising or, conversely, undertreating the older population.

\section{Author Contributions}

MJ writing - original draft and editing. AM and JA writing - review and editing.

\section{Funding}

The research was conducted during tenure of a Māori Health PhD Scholarship from the Health Research Council of New Zealand. MJ also received funding from the HOPE Foundation.

\section{Competing Interests}

The authors have declared that no competing interests exist.

\section{References}

1. World Health Organization. World report on ageing and health [Internet]. Geneva: WHO; 2015. Available from: https://www.who.int/ageing/events/world-report-2015-launch/en/. 
2. Lamster IB, Asadourian L, Del Carmen T, Friedman PK. The aging mouth: Differentiating normal aging from disease. Periodontol 2000. 2016; 72: 96-107.

3. Leonard R. Swallowing in the elderly: Evidence from fluoroscopy. Perspect Swollowing Disord. 2010; 19: 103-114.

4. Logemann JA, Curro FA, Pauloski B, Gensler G. Aging effects on oropharyngeal swallow and the role of dental care in oropharyngeal dysphagia. Oral Dis. 2013; 19: 733-737.

5. Gleeson DC. Oropharyngeal swallowing and aging: A review. J Commun Disord. 1999; 32: 373396.

6. Lee JW, Randall DR, Evangelista LM, Kuhn MA, Belafsky PC. Subjective assessment of videofluoroscopic swallow studies. Otolaryngol Head Neck Surg. 2017; 156: 901-905.

7. Yin $T$, Jardine $M$, Miles A, Allen J. What is a normal pharynx? A videofluoroscopic study of anatomy in older adults. Eur Arch Otorhinolaryngol. 2018; 275: 2317-2323.

8. Jardine $M$, Miles A, Allen J. A systematic review of physiological changes in swallowing in the oldest old. Dysphagia. 2020; 35: 509-532.

9. Allen JE, White CJ, Leonard RJ, Belafsky PC. Prevalence of penetration and aspiration on videofluoroscopy in normal individuals without dysphagia. Otolaryngol Head Neck Surg. 2010; 142: 208-213.

10. Namasivayam-MacDonald AM, Barbon CE, Steele CM. A review of swallow timing in the elderly. Physiol Behav. 2018; 184: 12-26.

11. Cock C, Omari T. Systematic review of pharyngeal and esophageal manometry in healthy or dysphagic older persons (>60 years). Geriatrics. 2018; 3: 67.

12. Jardine M, Miles A, Allen J, Leonard R. Quantifying post-swallow residue in healthy ageing. Perspectives of the ASHA Special Interest Groups. 2020; 5: 1657-1665.

13. Roy N, Stemple J, Merrill RM, Thomas L. Dysphagia in the elderly: Preliminary evidence of prevalence, risk factors, and socioemotional effects. Ann Otol Rhinol Laryngol. 2007; 116: 858865.

14. Namasivayam-MacDonald AM, Shune SE. The burden of dysphagia on family caregivers of the elderly: A systematic review. Geriatrics. 2018; 3: 30.

15. Ekberg O, Hamdy S, Woisard V, Wuttge-Hannig A, Ortega P. Social and psychological burden of dysphagia: Its impact on diagnosis and treatment. Dysphagia. 2002; 17: 139-146.

16. Belafsky PC, Mouadeb DA, Rees CJ, Pryor JC, Postma GN, Allen J, et al. Validity and reliability of the Eating Assessment Tool (EAT-10). Ann Otol Rhinol Laryngol. 2008; 117: 919-924.

17. Jardine M, Miles A, Allen J. Dysphagia onset in older adults during unrelated hospital admission: Quantitative videofluoroscopic measures. Geriatrics. 2018; 3: 66.

18. Leonard R. Predicting aspiration risk in patients with dysphagia: Evidence from fluoroscopy. Laryngoscope Investig Otolaryngol. 2019; 4: 83-88.

19. Bowling CB, Whitson HE, Johnson II TM. The 5Ts: Preliminary development of a framework to support inclusion of older adults in research. J Am Geriatr Soc. 2019; 67: 342-346.

20. Molnar FJ, Huang A, Tinetti M. Update: The public launch of the geriatric 5Ms [Internet]. Can Geriatr Soc J Contin Med Educ. Available from: http://canadiangeriatrics.ca/wpcontent/uploads/2017/04/UPDATE-THE-PUBLIC-LAUNCH-OF-THE-GERIATRIC-5MS.pdf. 
21. Jardine $M$, Miles $A$, Allen J. Self-reported swallowing and nutrition status in community-living older adults. Dysphagia. 2020. Doi: 10.1007/s00455-020-10125-y.

22. Binstock R. The oldest old: A fresh perspective or compassionate ageism revisited? Milbank Mem Fund Q Health Soc. 1985; 63: 420-451.

23. Kydd A, Fleming A, Paoletti I, Hvalič-Touzery S. Exploring terms used for the oldest old in the gerontological literature. J Aging Soc Chang. 2020; 10: 53-73.

24. Leder SB, Suiter DM, Agogo GO, Cooney LM. An epidemiologic study on ageing and dysphagia in the acute care geriatric-hospitalized population: A replication and continuation study. Dysphagia. 2016; 31: 619-625.

25. The World Bank. Life expectancy at birth, total (years) [Data file] [Internet]. 2018. Available from: https://data.worldbank.org/indicator/SP.DYN.LEOO.IN.

26. Kendall KA, Leonard RJ, McKenzie S. Common medical conditions in the elderly: Impact on pharyngeal bolus transit. Dysphagia. 2004; 19: 71-77.

27. Gnjidic D, Hilmer SN, Blyth FM, Naganathan V, Waite L, Seibel MJ, et al. Polypharmacy cutoff and outcomes: Five or more medicines were used to identify community-dwelling older men at risk of different adverse outcomes. J Clin Epidemiol. 2012; 65: 989-995.

28. Gallagher L, Naidoo P. Prescription drugs and their effects on swallowing. Dysphagia. 2009; 24: 159-166.

29. Ekberg O, Feinberg MJ. Altered swallowing function in elderly patients without dysphagia: Radiologic findings in 56 cases. Am J Roentgenol. 1991; 156: 1181-1184.

30. Soergel KH, Zboralske FF, Amberg JR. Presbyesophagus: Esophageal motility in nonagenarians. J Clin Invest. 1964; 43: 1472-1479.

31. Tack J, Vantrappen G. The aging oesophagus. Gut. 1997; 41: 422-424.

32. Fujishima I, Fujiu-Kurachi M, Arai H, Hyodo M, Kagaya H, Maeda K, et al. Sarcopenia and dysphagia: Position paper by four professional organizations. Geriatr Gerontol Int. 2019; 19: 9197.

33. Namasivayam-MacDonald AM, Riquelme LF. Presbyphagia to dysphagia: Multiple perspectives and strategies for quality care of older adults. Semin Speech Lang. 2019; 40: 227-242.

34. Walsh R. Meaning and purpose: A conceptual model for speech pathology terminology. Int J Speech Lang Pathol. 2005; 7: 65-76.

35. Feng $X$, Todd $T$, Lintzenich CR, Ding J, Carr JJ, Ge Y, et al. Aging-related geniohyoid muscle atrophy is related to aspiration status in healthy older adults. J Gerontol A Biol Sci Med Sci. 2012; 68: 853-860.

36. Baba T, Goto T, Fujimoto K, Honda T, Yagi K, Nagao K, et al. Age-related changes in geniohyoid muscle morphology predict reduced swallowing function. J Oral Heal Biosci. 2017; 30: 18-25.

37. Molfenter SM, Amin MR, Branski RC, Brumm JD, Hagiwara M, Roof SA, et al. Age-related changes in pharyngeal lumen size: A retrospective MRI analysis. Dysphagia. 2015; 30: 321-327.

38. Hara K, Tohara H, Kobayashi K, Yamaguchi K, Yoshimi K, Nakane A, et al. Age-related declines in the swallowing muscle strength of men and women aged $20-89$ years: A cross-sectional study on tongue pressure and jaw-opening force in 980 subjects. Arch Gerontol Geriatr. 2018; 78: 6470. 
39. Cruz-Jentoft AJ, Landi F, Topinková E, Michel JP. Understanding sarcopenia as a geriatric syndrome. Curr Opin Clin Nutr Metab Care. 2010; 13: 1-7.

40. Sakai K, Sakuma K. Frailty and sarcopenia-onset, development and clinical challenges. London: InTech; 2017. p. 81-102.

41. Wilson D, Jackson T, Sapey E, Lord JM. Frailty and sarcopenia: The potential role of an aged immune system. Ageing Res Rev. 2017; 36: 1-10.

42. Zhao WT, Yang M, Wu HM, Yang L, Zhang XM, Huang Y. Systematic review and meta-analysis of the association between sarcopenia and dysphagia. J Nutr Health Aging. 2018; 22: 1003-1009.

43. Leslie $P$, Drinnan MJ, Ford GA, Wilson JA. Swallow respiratory patterns and aging: Presbyphagia or dysphagia? J Gerontol A Biol Sci Med Sci. 2005; 60: 391-395.

44. de Lima Alvarenga EH, Dall'Oglio GP, Murano EZ, Abrahão M. Continuum theory: Presbyphagia to dysphagia? Functional assessment of swallowing in the elderly. Eur Arch Otorhinolaryngol. 2018; 275: 443-449.

45. Plowman EK, Humbert IA. Elucidating inconsistencies in dysphagia diagnostics: Redefining normal. Int J Speech Lang Pathol. 2018; 20: 310-317.

46. Kaspar K, Ekberg O. Identifying vulnerable patients: Role of the EAT-10 and the multidisciplinary team for early intervention and comprehensive dysphagia care. Nestle Nutr Inst Workshop Ser. 2012; 72: 19-31.

47. Hoogerduijn JG, Schuurmans MJ, Duijnstee MS, de Rooij SE, Grypdonck MF. A systematic review of predictors and screening instruments to identify older hospitalized patients at risk for functional decline. J Clin Nurs. 2007; 16: 46-57.

48. Smithard DG, Westmark S, Melgaard D. Evaluation of the prevalence of screening for dysphagia among older people admitted to medical services: An international survey. OBM Geriatr. 2019; 3: 8.

49. Adelman RD, Greene MG, Ory MG. Communication between older patients and their physicians. Clin Geriatr Med. 2000; 16: 1-24.

50. Chen PH, Golub JS, Hapner ER, Johns MM. Prevalence of perceived dysphagia and quality-oflife impairment in a geriatric population. Dysphagia. 2009; 24: 1-6.

51. Stewart K, Sen P. Pharyngeal pouch management: An historical review. J Laryngol Otol. 2016; 130: 116-120.

52. Jones B, Donner MW. How I do it: Examination of the patient with dysphagia. Dysphagia. 1989; 4: 162-172. 


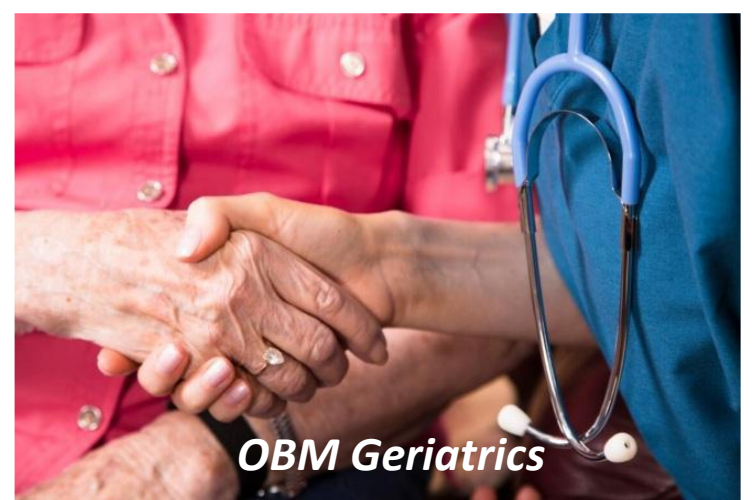

Enjoy $O B M$ Geriatrics by:

1. Submitting a manuscript

2. Joining in volunteer reviewer bank

3. Joining Editorial Board

4. Guest editing a special issue

For more details, please visit: http://www.lidsen.com/journals/geriatrics 